\title{
Evidências de Validade da Versão Brasileira do Job Crafting Questionnaire
}

\author{
Rita Pimenta de Devotto ${ }^{1,2}$ \\ Wagner de Lara Machado ${ }^{3}$ \\ ${ }^{1}$ Pontifícia Universidade Católica de Campinas \\ ${ }^{2}$ Faculdades de Campinas (Facamp), Campinas \\ ${ }^{3}$ Pontifícia Universidade Católica do Rio Grande do Sul (PUCRS)
}

\begin{abstract}
Resumo
A Escala de Ações de Redesenho do Trabalho é um instrumento de autorrelato para avaliação do job crafting, entendido como mudanças proativas conduzidas pelo indivíduo para modificar seu trabalho de forma a ajustá-lo às suas motivações, competências e valores. Suas propriedades psicométricas foram investigadas em dois estudos, sendo o primeiro com uma amostra on-line de 261 adultos e o segundo com 152 adultos, funcionários de uma organização privada do interior paulista. Os resultados de ambos os estudos corroboram a estrutura interna do instrumento por meio de técnicas exploratórias (análise fatorial exploratória e análise de rede) e análise fatorial confirmatória. O desempenho psicométrico da escala foi satisfatório em ambos os estudos no que diz respeito aos parâmetros de validade e fidedignidade. Observou-se a primazia da dimensão cognitiva do construto. Conclui-se que a Escala de Ações de Redesenho do Trabalho é uma medida válida e precisa no contexto brasileiro, possibilitando pesquisas futuras na área.

Palavras-chave: comportamento organizacional, avaliação psicológica, validade estatística, psicologia positiva
\end{abstract}

Validity Evidences of the Brazilian Version of the Job Crafting Questionnaire

\begin{abstract}
The Job Crafting Questionnaire is a self-report instrument for assessing job crafting, understood as proactive changes conducted by the individual to modify his own work to adjust it to his motivations, skills and values. Its psychometric properties were investigated in two studies, the first with an online sample of 261 adults and the second with 152 adults, employees of a private organization in the countryside of the state of São Paulo. The results of both studies supported the internal structure of the instrument by means of exploratory techniques (exploratory factor analysis and network analysis) and confirmatory factor analysis. The psychometric performance of the scale was satisfactory in both studies regarding the validity and reliability parameters. The primacy of the cognitive dimension of the construct was observed. We concluded that the Job Crafting Questionnaire is a valid and reliable instrument in the Brazilian context, enabling future research in the area.

Keywords: organizational behavior; psychological assessment; statistical validity; positive psychology.
\end{abstract}

\section{Evidencias de Validez de la Versión Brasileña del Job Crafting Questionnaire}

\begin{abstract}
Resumen
La Escala de Acciones de Rediseño del Trabajo es un instrumento de auto-informe para evaluación del job crafting, entendiéndose, como los cambios proactivos llevados a cabo por el individuo para modificar su trabajo con el fin de adaptarlo a sus motivaciones, habilidades y valores. Se investigaron sus propiedades psicométricas con dos estudios, el primero con una muestra online de 261 adultos y el segundo con 152, empleados de una organización privada del interior paulista. Los resultados de ambos estudios corroboraron la estructura interna del instrumento por medio de técnicas exploratorias (análisis factorial exploratorio y análisis de red) y análisis factorial confirmatorio. El desempeño psicométrico de la escala fue satisfactorio en ambos estudios, con respecto a los parámetros de validez y confiabilidad. Se observó la prioridad de la dimensión cognitiva del constructo. Se concluye que la Escala de Acciones de Rediseño del Trabajo es una medida válida y precisa en el contexto brasileño, posibilitando investigaciones futuras en el área.

Palabras clave: comportamiento organizacional; evaluación psicológica; validez estadística, psicología positiva
\end{abstract}

Nas últimas décadas, as mudanças nas economias globais têm alterado, em ritmo acelerado e de forma significativa, as características fundamentais do delineamento e organização do trabalho (Grant \& Parker, 2009). Tornou-se necessário superar as inadequações e ineficiências dos enfoques tradicionais "de cima para baixo" (top-down) (Demerouti, 2014; Demerouti \& Bakker, 2014), nos quais os gestores modificam o desenho e a organização do trabalho, visando aumentar tanto o desempenho como o bem-estar ocupacionais (Oldham \& Hackman, 2010).

Por outro lado, a ideia de que os trabalhadores realizam ações autodirigidas não planejadas para modificar suas funções e resolver problemas da organização, tem sido proposta e discutida em diversos estudos na literatura científica há alguns anos (Van Wingerden, Bakker, \& Derks, 2017). Recentemente, as perspectivas proativas (Grant \& Parker, 2009), enfatizaram a crescente 
importância dos funcionários para adaptar e introduzir mudanças na natureza do trabalho e para escolher os métodos para realizá-lo. As mudanças físicas e cognitivas que os indivíduos fazem nas tarefas ou nas relações de trabalho, em uma perspectiva "de baixo para cima" (bottom-up), foram denominadas de job crafting (Wrzesniewski \& Dutton, 2001). O "redesenho do trabalho" (Chinelato, Ferreira, \& Valentini, 2015) é a terminologia utilizada em português para fazer referência ao job crafting.

Indivíduos mais proativos tendem a modificar seu trabalho por meio de três tipos de estratégias que incluem: as ações de redesenho da tarefa (task crafting), as reformulações cognitivas (cognitive crafting) e as ações de redesenho das relações (relational crafting) (Wrzesniewski \& Dutton, 2001; Wrzesniewski, LoBuglio, Dutton, \& Berg, 2013). A primeira estratégia inclui mudanças materiais no conjunto de tarefas que o indivíduo considera ser seu trabalho (e.g., número, escopo e tipo de tarefa). As reformulações cognitivas envolvem a percepção do próprio trabalho, não apenas como um conjunto de tarefas concatenadas, mas como parte significativa de um todo. O terceiro tipo de ações engloba as mudanças na quantidade e na qualidade das interações no trabalho (com colegas, superiores, clientes, fornecedores).

Desde o surgimento do construto em 2001 até o ano de 2009, as pesquisas sobre job crafting foram essencialmente qualitativas ou de natureza teórica (Berg, Grant, \& Johnson, 2010a; Berg, Wrzesniewski, \& Dutton, 2010b; Wrzesniewski \& Dutton, 2001). Recentemente, duas escalas de job crafting para populações ocupacionais gerais foram validadas: a Job Crafting Scale - JCS (Tims, Bakker, \& Derks, 2012) e a Job Crafting Questionnaire - JCQ (Slemp \& Vella-Brodrick, 2013). Tims, Bakker e Derks (2012) operacionalizaram o construto dentro da perspectiva do modelo de Recursos e Demandas no Trabalho - RDT (Bakker \& Demerouti, 2007, 2013), propondo o job crafting como uma variável mediadora do engajamento no trabalho e da exaustão. As ações de redesenho do trabalho seriam funcionais para otimizar os recursos e as demandas do trabalho com as necessidades individuais e os recursos pessoais (Tims et al., 2012). Nessa perspectiva, o job crafting inclui quatro tipos de comportamentos, que visam: (1) aumentar os recursos estruturais do trabalho (e.g., autonomia), (2) aumentar os recursos sociais do trabalho (e.g., suporte dos colegas e supervisor) (3) aumentar as demandas de trabalho desafiadoras (e.g., novas responsabilidades) e (4) diminuir as demandas de trabalho consideradas como obstáculos (e.g., evitar relações de trabalho difíceis) (Tims et al., 2012).
A Job Crafting Scale foi validada em três estudos ( $N$ =1.181) conduzidos na Holanda (Tims et al., 2012). As análises fatoriais identificaram os quatro fatores teóricos, tendo as subescalas demonstrado índices adequados de fidedignidade: aumento dos recursos estruturais de trabalho (cinco itens, $\alpha=0,82$ ), aumento dos recursos sociais de trabalho (cinco itens, $\alpha=0,77$ ), aumento das demandas desafiadoras (cinco itens, $\alpha=0,75$ ), diminuição das demandas de obstáculo (seis itens, $\alpha=0,79$ ). Sobre as evidências de validade em relação às variáveis externas, o estudo evidenciou correlações positivas (baixa à moderada) com a personalidade proativa e a iniciativa pessoal; e correlações negativas com cinismo (dimensão do Burnout) e as três primeiras dimensões da Job Crafting Scale. A validade de critério foi averiguada em relação às avaliações de colegas sobre o engajamento e o desempenho no trabalho, evidenciando correlações positivas (Tims et al., 2012).

A versão brasileira da Job Crafting Scale foi nomeada Escala de Comportamentos de Redesenho no Trabalho -CRT (Chinelato et al., 2015). A CRT reuniu evidências de validade por meio de análises fatoriais confirmatórias, contudo, com a deleção da subescala Diminuição das demandas de obstáculo, por apresentar problemas na sua estrutura interna. Os autores argumentaram que dentre os fatores explicativos para a não replicabilidade da subescala, contribuíram a menor qualificação da amostra utilizada e as diferenças culturais nas relações de trabalho entre Brasil e Holanda. A versão final é composta de 14 itens distribuídos em três subescalas (aumento dos recursos estruturais, $\alpha=0,71$; aumento dos recursos sociais, $\alpha=0,78$; aumento das demandas desafiadoras, $\alpha=0,77)$. A CRT correlacionou de forma positiva com indicadores de engajamento no trabalho, com o desempenho intrapapel, com os afetos positivos no trabalho e com o capital psicológico positivo. Obteve-se também correlações negativas entre dimensões específicas e os indicadores de afeto negativo e neuroticismo (Chinelato et al., 2015).

O Job Crafting Questionnaire - JCQ (Slemp \& Vella-Brodrick, 2013) é uma alternativa para avaliação do construto, diferenciando-se da Job Crafting Scale por contemplar a dimensão cognitiva do construto. Além da dimensão de reformulações cognitivas (cognitive crafting, $\alpha=0,83$ ), o JCQ avalia as ações de redesenho da tarefa (task crafting, $\alpha=0,87$ ) e ações de redesenho das relações (relational crafting, $\alpha=0,89$ ) (Wrzesniewski \& Dutton, 2001). A análise da validade convergente corroborou evidências de que o JCQ foi positivamente correlacionado com índices de comportamento 
proativo (e.g., comportamento pró-social no trabalho e uso de forças positivas) e com o funcionamento positivo no trabalho (e.g., satisfação no trabalho, entusiasmo no trabalho e afeto positivo) e negativamente associado ao afeto negativo (Slemp \& Vella-Brodrick, 2013). Pelo conhecimento, apenas o estudo original foi conduzido com o objetivo de investigar o desempenho psicométrico da JCQ (Slemp \& Vella-Brodrick, 2013), sendo que estudos de validação da JCQ para outros idiomas são ainda inexistentes.

Portanto a operacionalização do construto em escalas fidedignas é um ganho recente. Esse fato tem influenciado a produção crescente de estudos empíri$\cos$ quantitativos na literatura internacional, em uma grande variedade de periódicos científicos de alto fator de impacto (Pimenta de Devotto \& Machado, 2017). Tendo em vista a crescente relevância da perspectiva do job crafting dentro da literatura internacional e o estágio ainda embrionário de pesquisas quantitativas sobre o construto no Brasil, justifica-se a validação de instrumentos fidedignos para nosso contexto. O presente estudo apresenta a adaptação e validação do JCQ para o Brasil. Buscou-se investigar as fontes de evidências de validade do JCQ baseadas na estrutura interna do instrumento, seguindo as diretrizes da literatura (American Educational Research Association [AERA], American Psychological Association [APA] \& National Councilon Measurement in Education [NCME], 2014). Foram conduzidos dois estudos com amostras independentes e empregados diferentes métodos de análise de dados (e.g., análise fatorial exploratória, analise fatorial confirmatória e análise de rede).

Estudo 1 - Estudo Exploratório da Versão Brasileira do Job Crafting Questionnaire

O Estudo 1 investigou de forma exploratória o desempenho psicométrico do JCQ. O desenho do estudo foi transversal e contou com uma amostra de profissionais acessados por meio de coleta em um survey on-line.

\section{Método}

\section{Adaptação do Job Crafting Questionnaire para o Brasil}

Procurando satisfazer as orientações gerais apresentadas na literatura (Borsa, Damásio, \& Bandeira, 2012; Muñiz, Elosua, \& Hambleton, 2013), foram seguidos passos sistemáticos para a adaptação do Job Crafting Questionnaire. Após o consentimento do autor do instrumento, três tradutores independentes e bilíngues conduziram a tradução do instrumento do inglês para o português brasileiro. Os autores deste artigo produziram uma versão sintetizada das três traduções. Posteriormente, dois especialistas bilíngues e com experiência na área de avaliação psicológica e psicologia organizacional, avaliaram a síntese das traduções independentes. Foram sugeridas mudanças na redação do enunciado em três dos 15 itens do instrumento. Após os ajustes indicados pelos juízes, um tradutor realizou o processo de retrotradução (back-translation). A versão retrotraduzida foi finalmente avaliada pelo autor do instrumento original. Após o seu consentimento, foi elaborada a versão final do instrumento, a Escala de Ações de Redesenho do Trabalho (EART). Essa versão foi submetida à aplicação assistida com seis profissionais de diferentes ocupações e níveis hierárquicos, sem revelar problemas de compreensão do instrumento.

\section{Validação da Escala de Ações de Redesenho do Trabalho}

\section{Participantes}

A amostra por foi composta por 261 trabalhadores, sendo 142 (54,8\%) do sexo feminino, $116(44,8 \%)$ do sexo masculino $(n=258)$. A média de idade foi de $45,22(D P=12,5)$ anos, com amplitude de 25 a $76(n$ = 261). Em relação à escolaridade, $164(63,3 \%)$ possuíam pós-graduação completa, $17(6,6 \%)$ possuíam pós-graduação incompleta, $65(25,1 \%)$ completaram o ensino superior, nove $(3,5 \%)$ declaram possuir ensino superior incompleto e apenas quatro $(1,6 \%)$ possuíam até o ensino médio completo $(n=259)$. Os participantes declararam residir em 14 estados brasileiros, sendo os principais São Paulo $(n=105 ; 40,5 \%)$, Minas Gerais $(n=95 ; 36,7 \%)$, Rio de Janeiro $(n=26 ; 10 \%)$ e Rio Grande do $\mathrm{Sul}(n=12 ; 4,6 \%)$. A responsabilidade por algum tipo de gestão (financeira, recursos, pessoas, projetos etc.) na função exercida foi declarada por 209 $(81,3 \%)$ participantes e $48(18,7 \%)$ afirmaram não ter nenhuma dessas responsabilidades em sua função. Os critérios de exclusão da amostra foram idade inferior a 25 anos e o fato do indivíduo ter menos de seis meses de trabalho na função. Devido aos dados não declarados, algumas informações apresentam variação em relação ao número total de participantes.

\section{Instrumentos}

Escala de Ações de Redesenho do Trabalho, EART (adaptação do Job Crafting Questionnaire JCQ, Slemp \& Vella-Brodrick, 2013). O instrumento é composto por 15 itens, respondidos em uma escala tipo Likert de seis pontos, na qual os extremos são 
"raramente" (1) e "com muita frequência" (6). O participante indica a frequência com que ele se engaja em quinze ações diferentes de job crafting. A escala é dividida em três subescalas (cada uma com cinco itens) que avaliam as ações de redesenho na tarefa (task crafting) e nas relações (relational crafting) e as reformulações cognitivas (cognitive crafting). Exemplos de itens: "Muda a abrangência (escopo) ou os tipos de tarefas que você realiza no trabalho" (tarefa), "Organiza ou frequenta atividades sociais relacionadas com seu trabalho" (relações) e "Pensa em como seu trabalho lhe traz sentido e propósito na vida" (cognitiva). Slemp e Vella-Brodrick (2013) reportam índices de fidedignidade adequados para as três as subescalas (task crafting: $\alpha=0,87$; cognitive crafting: $\alpha=0,89$; relacional crafting: $\alpha=0,83$ ) e para a escala global $(\alpha=0,91)$. As medidas de fidedignidade das subescalas, no presente estudo, serão descritas na seção "Resultados".

Questionário sociodemográfico. O instrumento identifica a amostra do estudo em relação às variáveis demográficas relevantes para a pesquisa. Foram questionadas informações sobre o sexo, a idade, o nível educacional, a região de residência, dentre outras informações.

\section{Procedimentos}

A coleta de dados foi realizada por meio de um questionário on-line, na plataforma Survey Monkey, divulgado em redes sociais e profissionais. O sigilo e a confidencialidade sobre a identidade dos participantes foram garantidos, conforme constava no Termo de Consentimento Livre e Esclarecido, segundo as diretrizes da resolução 510/2016 e anteriores do Conselho Nacional de Saúde. Esta pesquisa recebeu parecer favorável do Comitê de Ética em Pesquisa, sob o $n^{\circ}$ CAAE: 49694115.0.0000.5481 em novembro de 2015.

\section{Análise de Dados}

Seguindo as diretrizes da literatura (AERA, APA \& NCME, 2014), a estratégia de validação empregou várias técnicas para estimar os parâmetros psicométricos e a dimensionalidade do instrumento, contribuindo para agregar evidências de sua validade no contexto brasileiro. Primeiramente foi conduzida uma análise paralela (Timmerman \& Lorenzo-Seva, 2011), como método de retenção de fatores para testar a replicabilidade do modelo de três fatores (Slemp \& Vella-Brodrick, 2013). Esse tipo de análise permite estabelecer o número de fatores e avaliar o poder de explicação dos dados comparado à permutação dos valores amostrais e matrizes aleatórias. Foi utilizado o software R e o pacote Psych (Revelle, 2016). Valores faltantes (missing values) representaram menos de 1\% dos dados, e nenhum procedimento de imputação foi adotado.

Posteriormente, empreendeu-se uma análise fatorial exploratória com método de extração Ordinary Least Squares, com rotação Oblimin, para averiguar o livre agrupamento de itens nos três fatores retidos. A adequação de ajuste da EART foi avaliada por meio dos seguintes índices Tuker-Lewis Index (TLI $\geq 0,90)$, e Root Mean Square Error of Approximation (RMSEA $\leq 0,08$, com intervalo de confiança de 95\%) (Brown, 2006). Os itens foram interpretados nos fatores a partir das cargas fatoriais $\leq 0,30$. A consistência interna foi avaliada utilizando o coeficiente de fidedignidade alfa de Cronbach ordinal (Gadermann, Guhn, \& Zumbo, 2012). Valores superiores a 0,70 são considerados satisfatórios e valores superiores a 0,90 indicam alta confiabilidade.

Em seguida, empreendeu-se uma análise de rede (Borsboom \& Cramer, 2013; Schmittmann et al., 2011) para investigar o padrão de relações entre os itens da EART. Diferentemente da análise fatorial que visa identificar variáveis latentes que explicam as covariâncias entre os itens de um instrumento, a análise de rede é um modelo exploratório baseado diretamente na interação par-a-par entre elementos em um sistema (Machado, Vissoci, \& Epskamp, 2015), no caso, os itens da EART. As relações (arestas) entre os itens (vértices) são representadas em um grafo bidimensional, no qual as variáveis são aproximadas ou expelidas com base no peso das suas associações (Fruchterman \& Reingold, 1991)

Foram estimados os grafos de correlação bivariada e de correlações parciais por meio do algoritmo Graphical Least Abssolute Shrinkage and Selection Operator - GeLASSO (Friedman, Hastie, \& Tibshirani, 2008), no qual as relações par-a-par entre os itens são condicionadas (i.e., controladas estatisticamente) aos demais itens. Dessa forma, obtém-se um grafo esparso, representando apenas as relações mais relevantes no sistema. Para evitar o super ajuste do modelo aos dados, a solução final é obtida após uma penalização que fixa em zero as correlações parciais de pequena magnitude, por meio do índice Extended Bayesian Information Criterion (EBIC; Chen \&Chen, 2008). Posteriormente, buscou-se identificar subagrupamentos (que possuem maior conexões entre seus itens e menores conexões com itens de outros agrupamentos) de itens no grafo de correlações parciais por meio de análises de comunidades. Os algoritmos utilizados foram Walktrap (Pons \& Latapy, 2005), Spinglass 
(Reichardt \& Bornholdt, 2006) e Lowvain (Blondel, Guillaume, Lambiotte, \& Lefebvre, 2008).

Por fim, estatísticas descritivas da rede (também chamadas de medidas de centralidade) de correlações parciais foram obtidas para melhor representar o papel de cada item (comportamento) no conjunto do construto. As estatísticas referem-se ao número de conexões que perpassam um vértice (conectividade), a conectividade de um vértice com outros no sistema (proximidade) e a soma modular do peso de associações de um vértice com outros no sistema (força). Essas análises foram conduzidas por meio do software $\mathrm{R}$ e do pacote Pgraph (Epskamp, Cramer, Waldorp, Schmittmann, \& Borsboom, 2012).

\section{Resultados}

A análise paralela (Timmerman \& Lorenzo-Seva, 2011) demonstrou que a solução de três fatores é adequada à escala, corroborando os pressupostos teóricos do construto do job crafting (Slemp \& Vella-Brodrick, 2013; Wrzesniewski \& Dutton, 2001). O poder de explicação da estrutura de três dimensões da amostra é superior àquela esperada ao acaso (dados simulados). Portanto na análise fatorial exploratória, o número de fatores foi limitado a três. Nesse modelo, no qual foi investigada a solução multifatorial de três fatores de primeira ordem, obteve-se índices de ajuste bastante satisfatórios: TLI $(=0,948)$ e resíduos nos patamares aceitáveis RMSEA (90\% IC) = 0,063 (0,045 - 0,077). Todos os itens apresentaram carga fatorial adequada em suas respectivas dimensões (Tabela 1), conforme proposto teoricamente (Slemp \& Vella-Brodrick, 2013), à exceção do item cinco, pertencente à dimensão da tarefa no modelo original. Esse item apresentou carga cruzada, sendo explicado tanto na dimensão da tarefa $(\lambda=0,30)$, como na dimensão cognitiva $(\lambda=0,28)$. Nesse modelo resultante da análise fatorial exploratória, os fatores estiveram significativamente associados e seus índices de consistência interna foram satisfatórios (Tabela 1).

A análise de comunidades, como indicado na Figura 1, também corroborou a estrutura de agrupamento de itens encontrada na análise fatorial exploratória. Dessa forma, a análise corrobora $\mathrm{O}$ modelo original da escala, destacando que o item cinco permaneceu na dimensão tarefa.

As medidas de centralidade da análise de rede (Figura 2) também ajudaram a explicar a carga cruzada do item cinco, encontrada na análise fatorial exploratória. É possível observar que o item seis funcionou como um conector entre as dimensões cognitiva e da tarefa, e exerceu forte influência no item cinco.

Estudo 2 - Análise Confirmatória da Escala de Ações de Redesenho do Trabalho

O Estudo 2 investigou por meio de análises fatoriais confirmatórias a adequação da estrutura de três dimensões da EART encontrada no Estudo 1. Para isso, foram analisados os dados de uma amostra independente de profissionais de uma organização privada do interior paulista.

\section{Método}

\section{Participantes}

A amostra por conveniência do Estudo 2 foi composta por 152 trabalhadores, sendo $99(65,1 \%)$ do sexo feminino, $52(34,2 \%)$ do sexo masculino, e um participante não informou $(0,7 \%)$. A média de idade foi de 42,30 (DP =12,11) anos, com amplitude de 25 a 78. Residiam no estado de São Paulo 149 (98\%) participantes e apenas três, em outras regiões do país. Em relação à escolaridade, $68(44,7 \%)$ possuíam pós-graduação completa, nove $(5,9 \%)$ possuíam pós-graduação incompleta, $56(36,8 \%)$ completaram o ensino superior, 11 $(7,2 \%)$ declaram possuir ensino superior incompleto, sete $(4,6 \%)$ possuíam até o ensino médio completo e um participante $(0,7 \%)$ completou o ensino fundamental. A responsabilidade por algum tipo de gestão (financeira, recursos, pessoas, projetos, etc.) na função exercida foi declarada por $78(51,3 \%)$ participantes e 74 $(48,7 \%)$ disseram que sua função não envolvia nenhum tipo de gestão. Os critérios de exclusão da amostra foram idade inferior a 25 anos e o fato do indivíduo ter menos de seis meses de trabalho na função. Devido aos dados não declarados, algumas informações apresentam variação em relação ao número total de sujeitos. Instrumentos

Foi utilizada a Escala de Ações de Redesenho no Trabalho e o mesmo questionário sociodemográfico utilizado no Estudo 1.

\section{Procedimentos}

Os procedimentos éticos foram idênticos ao do Estudo 1, porém o procedimento de coleta de dados foi realizado por meio de um questionário on-line, na plataforma Survey Monkey. O instrumento foi divulgado pelo setor de Recursos Humanos de uma organização privada no Estado de São Paulo, para todos os seus funcionários, por meio do serviço de e-mail. 
Tabela 1.

Resultados da Análise Fatorial Exploratória e Confiabilidade

\begin{tabular}{|c|c|c|c|c|}
\hline Itens (conteúdo resumido) & $\begin{array}{l}\text { Fator } 1 \\
\text { Tarefa }\end{array}$ & $\begin{array}{c}\text { Fator } 2 \\
\text { Cognitivo }\end{array}$ & $\begin{array}{c}\text { Fator } 3 \\
\text { Relações }\end{array}$ & Comunalidade \\
\hline 1. Faz de forma diferente. & 0,73 & 0,09 & $-0,02$ & 0,61 \\
\hline 2. Muda a abrangência e tipos de tarefa. & 0,83 & $-0,06$ & $-0,02$ & 0,62 \\
\hline $\begin{array}{l}\text { 3. Novas tarefas que se adequam às } \\
\text { habilidades e preferências. }\end{array}$ & 0,79 & 0,01 & 0,00 & 0,65 \\
\hline 4. Assume tarefas adicionais. & 0,59 & 0,10 & 0,10 & 0,51 \\
\hline $\begin{array}{l}\text { 5. Dá preferência às tarefas que se adequam } \\
\text { às habilidades. }\end{array}$ & 0,30 & 0,28 & 0,05 & 0,31 \\
\hline 6. Trabalho traz sentido e propósito. & 0,18 & 0,67 & 0,09 & 0,71 \\
\hline $\begin{array}{l}\text { 7. Significado do próprio trabalho para } \\
\text { sucesso da organização. }\end{array}$ & 0,09 & 0,69 & 0,08 & 0,64 \\
\hline $\begin{array}{l}\text { 8. Importância do trabalho para a } \\
\text { comunidade de inserção. }\end{array}$ & 0,13 & 0,62 & 0,08 & 0,58 \\
\hline 9. Trabalho influencia positivamente a vida. & $-0,06$ & 0,96 & $-0,05$ & 0,81 \\
\hline 10. Papel do trabalho no bem-estar geral. & $-0,03$ & 0,83 & 0,00 & 0,66 \\
\hline 11. Conhecer bem as pessoas no trabalho. & 0,02 & 0,19 & 0,54 & 0,45 \\
\hline 12. Organiza e frequenta atividades sociais. & $-0,01$ & 0,04 & 0,69 & 0,50 \\
\hline 13. Organiza eventos especiais no trabalho. & $-0,04$ & $-0,08$ & 0,81 & 0,58 \\
\hline 14. Mentor de novos empregados. & 0,16 & $-0,06$ & 0,59 & 0,40 \\
\hline 15. Amizades com pessoas no trabalho. & $-0,05$ & 0,14 & 0,59 & 0,42 \\
\hline \multicolumn{5}{|l|}{ Correlações } \\
\hline F2 (Cognitivo) & 0,67 & & & \\
\hline F3 (Relações) & 0,44 & 0,51 & & \\
\hline Variância explicada & 0,32 & 0,41 & 0,28 & \\
\hline Fidedignidade ( $\alpha$ ordinal) & 0,82 & 0,90 & 0,79 & \\
\hline
\end{tabular}

Análise de Dados

Com o objetivo de confirmar a estrutura de três fatores da EART encontrada no Estudo 1, realizou-se uma análise fatorial confirmatória para verificar os ajustes do modelo teórico do job crafting composto de três fatores (Slemp \& Vella-Brodrick, 2013) aos dados coletados na amostra de profissionais de uma organização privada do Estado de São Paulo. Foram considerados os índices de Tuker-Lewis Index (TLI $\geq 0,90$ ) e Root mean square error of approximation (RMSEA $\leq 0,08$, com intervalo de confiança de 95\%) (Brown, 2006; Jackson, Gillaspy Jr., \& Purc-Stephenson, 2009). As análises foram conduzidas por meio do software $\mathrm{R}$ e do pacote Lavaan (Rosseel, 2012). Valores faltantes (missing values) representaram menos de $1 \%$ dos dados e nenhum procedimento de imputação foi adotado.

\section{Resultados}

A análise fatorial confirmatória (AFC) do Estudo 2 confirmou a estrutura encontrada no Estudo 1, ou seja, a existência de três fatores significativamente associados e cujo agrupamento de itens corrobora o modelo original (Slemp \& Vella-Brodrick, 2013). Os índices de ajuste foram satisfatórios: $c^{2}(87)=108,47 ; p=0,06$, TLI $=0,93$ e resíduos nos patamares aceitáveis RMSEA $(90 \%$ IC $)=0,042(0,000-0,065)$. Todos os itens apresentaram carga fatorial adequada em suas respectivas dimensões, conforme proposto teoricamente (Slemp \& Vella-Brodrick, 2013) e os três fatores estiveram significativamente associados (Figura 3). Os índices de fidedignidade composta (FC) das três subescalas foram satisfatórios $(0,80 ; 0,93$ e 0,75 para as subescalas Tarefa, 


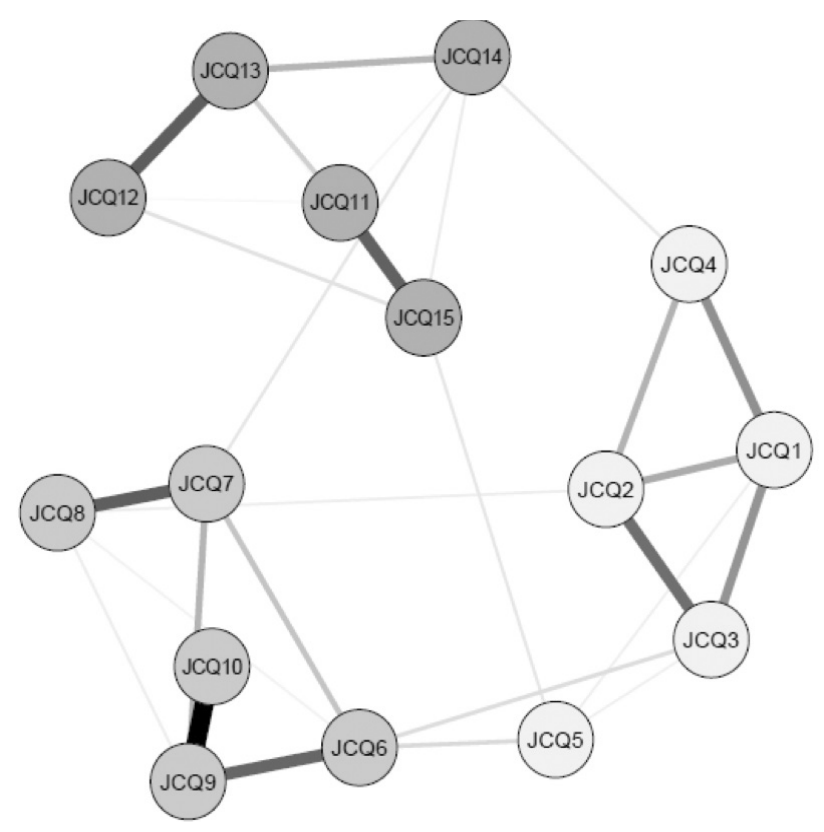

Figura 1. Análise de rede e comunidade dos itens do JCQ. A análise permitiu identificar os agrupamentos de itens correspondentes aos fatores originais do instrumento, sendo: redesenho da tarefa (cinza claro), reformulação cognitiva (cinza médio) e redesenho das relações (cinza escuro).

Cognitiva e Relações, respectivamente). Da mesma forma, a variância média extraída dos itens de cada um dos fatores foi respectivamente tarefa $=45 \%$, cognitiva $=73 \%$ e relações $=39 \%$.

\section{Discussão}

A presente pesquisa teve como objetivo adaptar e reunir evidências de validade da estrutura interna da Escala de Ações de Redesenho do Trabalho, em duas amostras de profissionais distintas e valendo-se de diferentes técnicas de análise de dados. Os Estudos 1 e 2 revelaram que a estrutura de três fatores de primeira ordem do instrumento original é replicada para o contexto brasileiro, corroborando as proposições teóricas do construto (Wrzesniewski \& Dutton, 2001) e de sua avaliação (Slemp \& Vella-Brodrick, 2013).

A análise de comunidades e as medidas de centralidade revelaram o papel fundamental do item seis da EART, o qual remete à frequência com que o trabalhador "pensa em como o seu trabalho lhe traz sentido e propósito de vida". O item seis surgiu como um nodo central (Figura 1), o qual apresentou relações de grande

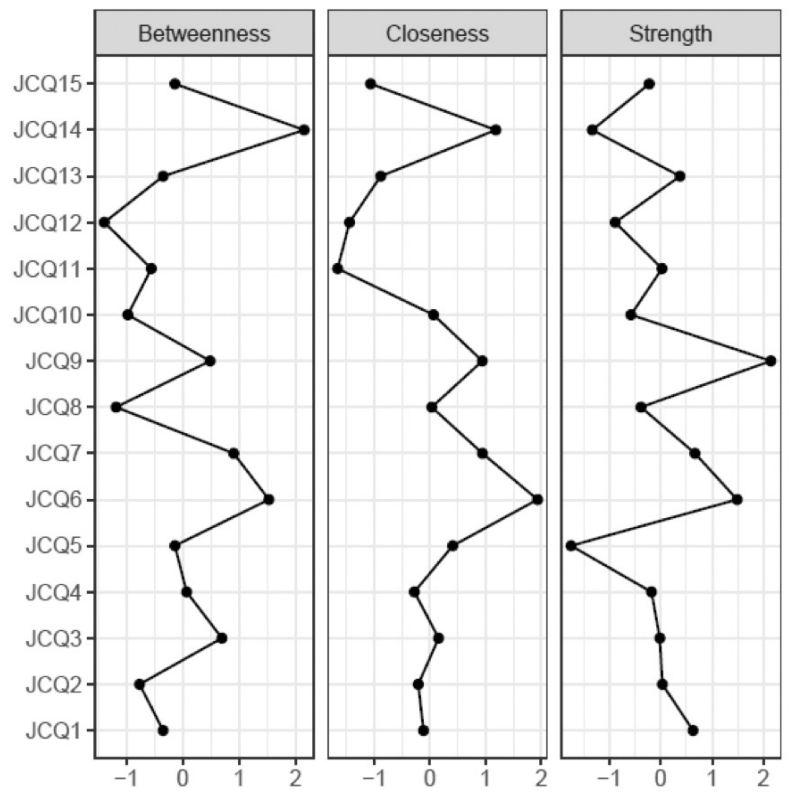

Figura 2. Medidas de centralidade dos itens da Escala de Ações de Redesenho do Trabalho. Betweeness = conectividade; Closeness $=$ proximidade; Strenght $=$ força.

magnitude com os demais nodos do sistema. As medidas de centralidade (Figura 2) revelaram que o item seis apresentou um elevado grau de proximidade, ou seja, um maior número de conexões com todos os demais itens. Seu grau de conectividade foi expressivo, indicando ser esse o caminho mais curto (elo) entre todos os pares de dados conectados na rede. A força do item seis indicou seu poder de estabelecer relações de maior magnitude com outros itens.

O posicionamento e força do item seis da EART permitiu entender a carga cruzada do item cinco na análise fatorial exploratória, o qual foi explicado tanto pela dimensão da tarefa (à qual ele pertence), como pela dimensão cognitiva. O item cinco "Dá preferência às tarefas de trabalho que se adequam às suas habilidades ou interesses" foi fortemente influenciado pelo item seis, o qual faz referência à percepção do indivíduo sobre o sentido e propósito do trabalho na vida. A posição do item seis como um nodo que espalha influência em todo o sistema poderia ser explicada como uma cognição saliente que estaria antecipando as demais ações de redesenho do trabalho. Portanto, apresentam-se evidências de que o redesenho do trabalho compreende um conjunto variado de ações que incluem não apenas mudanças tangíveis nas tarefas e nas relações, mas também mudanças na forma de perceber o trabalho, o que 


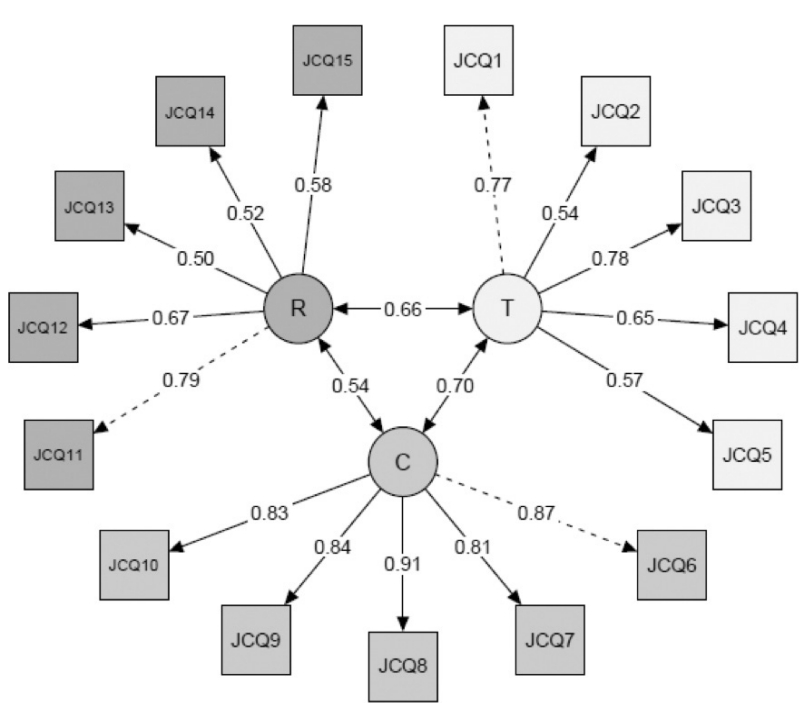

Figura 3. Análise fatorial confirmatória da Escala de Ações de Redesenho do Trabalho.

por sua vez pode influenciar novas ações de redesenho das tarefas e das relações.

A dimensão cognitiva esteve omissa nas escalas anteriores que buscaram avaliar o construto na perspectiva teórica do modelo de recursos e demandas no trabalho (Slemp \& Vella-Brodrick, 2013). Nossos achados corroboram para o fato de que a dimensão cognitiva é a mais saliente do construto, dado sua elevada consistência interna na amostra dos Estudos $1 \mathrm{e}$ 2 ( $\alpha=0,90$ e $\alpha=0,93$, respectivamente) e primazia na extração dos fatores. A dimensão cognitiva apresentou também maior variância explicada $(16,81 \%)$ em relação às respostas da amostra do Estudo 1, quando comparada às dimensões da tarefa $(10 \%)$ e das relações (7,84\%). Esse mesmo padrão também foi observado na análise fatorial confirmatória do Estudo 2, no qual a dimensão cognitiva explicou $73 \%$ da variância dos seus itens, comparado com a dimensão da tarefa (45\%) e com a dimensão das relações (39\%).

As reformulações cognitivas (cognitive crafting) alteram a percepção de que o indivíduo tem do próprio trabalho para torná-lo pessoalmente significativo e gratificante (Slemp \& Vella-Brodrick, 2013). Sugere-se que a dimensão cognitiva seja a que mais estreitamente se alinhe ao conceito de identidade no trabalho (Slemp \& Vella-Brodrick, 2013), porque, ao mudar a forma como o trabalho é visto, os indivíduos moldam sua experiência de trabalho (Wrzesniewski \& Dutton, 2001), influenciando novas ações de redesenho nas tarefas e nas relações de trabalho e apreciando os efeitos mais amplos que seu trabalho pode exercer na organização e na comunidade.

O modelo teórico propõe que o job crafting acontece em diferentes âmbitos laborais, independentemente do grau de autonomia, complexidade e autoridade envolvido no trabalho (Wrzesniewski \& Dutton, 2001; Wrzesniewski et al., 2013). Além da existência de diferenças amostrais nos diversos critérios sociodemográficos, destaca-se a marcante diferença com relação à responsabilidade de gestão imbuída na função. $\mathrm{Na}$ amostra do Estudo 1, 81,3\% dos participantes tinham responsabilidade por algum tipo de gestão na função exercida, enquanto, no Estudo 2, 48,7\% dos participantes realizavam trabalhos nos quais não havia responsabilidade de gestão. Essa diferença ilustra o fato de que o job crafting se faz presente nos trabalhos rotineiros e naqueles mais complexos e em diferentes níveis hierárquicos de uma organização (Berg, Wrzesniewski, \& Dutton, 2010b).

\section{Considerações Finais}

A inclusão da dimensão cognitiva na avaliação do job crafting é um ganho importante, porque permitirá avaliar a sequência temporal das ações de job crafting (Slemp \& Vella-Brodrick, 2013). Uma hipótese é de que as mudanças na forma de perceber o trabalho (cognitive crafting) precedam os comportamentos de redesenho do trabalho que afetam a dimensão da tarefa (e.g., aumento dos recursos estruturais, aumento das demandas desafiadoras e diminuição das demandas de obstáculo) e aqueles que compõem as mudanças nas relações (e.g., aumento dos recursos sociais).

Entre os pontos fortes desta investigação, destaca-se a utilização de duas amostras diferentes e de diversos procedimentos de análise de dados. Em razão da heterogeneidade da amostra total utilizada, os resultados sugerem que a EART é um instrumento adequado para avaliar o construto em diferentes contextos de trabalho no Brasil. Essa afirmação deverá ser corroborada com uma amostra representativa da população brasileira em pesquisas futuras, visando a construção de normas para a EART. As limitações deste estudo referem-se ao uso de uma amostra por conveniência e ao fato de não ter sido investigado a validade do instrumento por meio da associação com variáveis externas.

O presente artigo contribui ao apresentar evidências iniciais de validade da EART para o contexto brasileiro. A sua utilização em pesquisas futuras pode contribuir para a avaliação da proatividade do indivíduo 
no desenvolvimento dos processos de trabalho, para o entendimento das questões relativas ao significado e identidade no trabalho, bem como para uma avaliação da influência dos três tipos de estratégias de job crafting em diferentes variáveis de desfecho (e.g., desempenho no trabalho, saúde mental positiva).

\section{Referências}

American Educational Research Association, American Psychological Association, \& National Council on Measurement in Education (2014). Standards for educational and psychological testing. Washington, DC: American Educational Research Association.

Bakker, A. B., \& Demerouti, E. (2007). The Job Demands-Resources model: State of the art. Journal of Managerial Psychology, 22(3), 309-328. doi: 10.1108/02683940710733115

Bakker, A. B., \& Demerouti, E. (2013). La teoría de las demandas y los recursos laborales. Revista de Psicología del Trabajo y de las Organizaciones, 29(3), 107-115. doi: doi.org/10.5093/tr2013a16

Berg, J. M., Grant, A. M., \& Johnson, V. (2010a). When callings are calling: Crafting work and leisure in pursuit of unanswered occupational callings. Organization Science, 21(5), 973-994. doi: 10.1287/ orsc. 1090.0497

Berg, J. M., Wrzesniewski, A., \& Dutton, J. E. (2010b). Perceiving and responding to challenges in job crafting at different ranks: When proactivity requires adaptivity. Journal of Organizational Behavior, 31(2-3), 158-186. doi:10.1002/job.645

Blondel, V. D., Guillaume, J. L., Lambiotte, R., \& Lefebvre, E. (2008). Fast unfolding of communities in large networks. Journal of statistical mechanics: Theory and experiment, 2008(10). doi: 10.1088/1742-5468/2008/10/P10008

Borsboom, D., \& Cramer, A. O. (2013). Network analysis: An integrative approach to the structure of psychopathology. Annual review of clinical psychology, 9, 91-121. doi: 10.1146/ annurev-clinpsy-050212-185608

Borsa, J. C., Damásio, B. F., \& Bandeira, D. R. (2012). Cross-cultural adaptation and validation of psychological instruments: Some considerations. Paidéia (Ribeirão Preto), 22(53), 423-432. doi: 10.1590/1982-43272253201314
Brown, T. A. (2006). Confirmatory factor analysis for applied research. New York: The Guilford Press.

Chen, J., \& Chen, Z. (2008). Extended Bayesian information criteria for model selection with large model spaces. Biometrika, 95, 759-771. doi: 10.1093/biomet/asn034

Chinelato, R. S. D. C., Ferreira, M. C., \& Valentini, F. (2015). Evidence of Validity of the Job Crafting Behaviors Scale. Paidéia (Ribeirão Preto), 25(62), 325332. doi: 10.1590/1982-43272562201506

Demerouti, E. (2014). Design your own job through job crafting. European Psychologist, 19(4), 237-247. doi: 10.1027/1016-9040/a000188

Demerouti, E., \& Bakker, A. B. (2014). Job crafting. Em M. C. W. Peeters, J. de Jonge, \& T. W. Taris (Org.), An introduction to contemporary work psychology (pp. 414-433). New York, NY: Wiley-Blackwell.

Epskamp, S., Cramer, A. O., Waldorp, L. J., Schmittmann, V. D., \& Borsboom, D. (2012). Qgraph: Network visualizations of relationships in psychometric data. Journal of Statistical Software, 48(4), 1-18. doi: $10.18637 /$ jss.v048.i04

Friedman, J., Hastie, T., \& Tibshirani, R. (2008). Sparse inverse covariance estimation with the graphical lasso. Biostatistics, 9(3), 432-441. doi: 10.1093/biostatistics/kxm045

Fruchterman, T. M. J., \& Reingold, E. M. (1991). Graph drawing by force-directed placement. Software Practice and Experience, 12, 1129-1164. doi: 10.1002/ spe.4380211102

Gadermann, A. M., Guhn, M., \& Zumbo, B. D. (2012). Estimating ordinal reliability for Likert-type and ordinal item response data: A conceptual, empirical, and practical guide. Practical Assessment, Research \& Evaluation, 17(3), 1-13. Recuperado de http:// pareonline.net/getvn.asp?v $=17 \& n=3$

Grant, A. M., \& Parker, S. K. (2009). Redesigning work design theories: The rise of relational and proactive perspectives. The Academy of Management Annals, 3(1),317-375. doi: 10.1080/19416520903047327

Jackson, D. L., Gillaspy Jr, J. A., \& Purc-Stephenson, R. (2009). Reporting practices in confirmatory factor analysis: An overview and some recommendations. Psychological Methods, 14(1), 6.doi: 10.1037/ a0014694 
Machado; W. L., Vissoci, J. R. N., \& Epskamp, S. (2015). Análise de rede aplicada à psicometria e à avaliação psicológica. Em C. S. Hutz, D. R. Bandeira, \& C. M. Trentini, (Org.). Psicometria (pp. 125-146). Porto Alegre: Artmed.

Muñiz, J., Elosua, P., \& Hambleton, R. K. (2013). Directrices para la traducción y adaptación de los tests: Segunda edición. Psicothema, 25(2), 151-157. doi: 10.7334/psicothema2013.24

Oldham, G. R., \& Hackman, J. R. (2010). Not what it was and not what it will be: The future of job design research. Journal of organizational behavior, 31(2-3), 463-479. doi: 10.1002/job.678

Pimenta de Devotto, R., \& Machado, W.L. (2017). Job crafting: Uma revisão da literatura internacional. Psico-USF, 22(3), 413-423. doi: 10.1590/1413-82712017220303

Pons, P., \& Latapy, M. (2005). Computing communities in large networks using random walks. Em International symposium on computer and information sciences (pp. 284-293). Springer, Berlin, Heidelberg.

Reichardt, J., \& Bornholdt, S. (2006). Statistical mechanics of community detection. Physical Review E, 74(1), 016-110. doi:10.1103/PhysRevE.74.016110

Revelle, W. (2018). Psych: Procedures for Personality and Psychological Research. Northwestern University: Evanston.

Rosseel, Y. (2012). Lavaan: An R Package for Structural Equation Modeling. Journal of Statistical Software, 48(2), 1-36. doi:10.18637/jss.v048.i02

Schmittmann, V. D., Cramer, A. O., Waldorp, L. J., Epskamp, S., Kievit, R. A., \& Borsboom, D. (2013). Deconstructing the construct: A network perspective on psychological phenomena. New Ideas in Psychology, 31(1), 43-53. doi: 10.1016/j. newideapsych.2011.02.007
Slemp, G. S., \& Vella-Brodrick, D. A. (2013). The job crafting questionnaire: A new scale to measure the extent to which employees engage in job crafting. International Journal of Wellbeing, 3(2), 126-146. doi: 10.5502/ijw.v3i2.1

Tims, M., \& Bakker, A. B. (2010). Job crafting: Towards a new model of individual job redesign. South African Journal of Industrial Psychology, 36(2), 1-9. doi: 10.4102/sajip.v36i2.841

Tims, M., Bakker, A. B., \& Derks, D. (2012). Development and validation of the job crafting scale. Journal of Vocational Behavior, 80(1), 173-186. doi: 10.1016/j.jvb.2011.05.009

Timmerman, M. E., \& Lorenzo-Seva, U. (2011). Dimensionality assessment of ordered polytomous items with parallel analysis. Psychological Methods, 16(2), 209-220. doi: 10.1037/a0023353

Van Wingerden, J., Bakker, A. B., \& Derks, D. (2017). The longitudinal impact of a job crafting intervention. European Journal of Work and Organizational Psychology, 26(1), 107-119. doi: 10.1080/1359432X.2016.1224233

Wrzesniewski, A., \& Dutton, J. E. (2001). Crafting a job: Revisioning employees as active crafters of their work. Academy of Management Review, 26(2), 179-201. doi: 10.5465/AMR.2001.4378011

Wrzesniewski, A., LoBuglio, N., Dutton, J. E., \& Berg, J. M. (2013). Job crafting and cultivating positive meaning and identity in work. Em A. B. Bakker (Org.), Advances in positive organizational psychology (pp. 281-302). UK: Emerald Publishing Group.

Recebido em: 28/09/2017 Reformulado em: 25/07/2018, 23/09/2018, 30/11/2018

Aceito: 22/01/2019 
Sobre os autores:

Rita Pimenta de Devotto é pedagoga (Unicamp), com MBA Executivo pelo IAE Business School (Argentina), mestre em Creativity and Problem Solving, State University of New York (EUA), mestre em Psicologia (PUCAMP), doutoranda do Programa de Pós-Graduação em Psicologia da PUC - Campinas e Professora do curso de Administração de Empresas da Faculdades de Campinas (Facamp).

ORCID: https://orcid.org/0000-0002-2287-2526

E-mail: rita.pimenta@facamp.com.br

Wagner de Lara Machado é psicólogo (ULBRA), doutor em Psicologia (UFRGS), com pós-doutorado em Psicometria e Métodos Quantitativos (UFRGS), professor permanente do programa de pós-graduação stricto sensu em Psicologia da Pontifícia Universidade Católica do Rio Grande do Sul (PUCRS), coordenador do grupo de pesquisa Avaliação em Bem-estar e Saúde Mental (ABES).

ORCID: https://orcid.org/0000-0001-5555-5116

E-mail:wagner.machado@pucrs.br

Contato com os autores:

Rita Pimenta de Devotto

Av. Alan Turing, 805, Cidade Universitária

Campinas-SP, Brasil

CEP: 13083-898

Psico-USF, Bragança Paulista, v. 25, n. 1, p. 39-49, jan./mar. 2020 